\title{
On the rationality of the singularity locus of a Gough-Stewart platform - biplanar case
}

\author{
Michel Coste* $^{*} \quad$ Seydou Moussa ${ }^{\dagger}$
}

July 3, 2014

\begin{abstract}
We propose to study the singularity locus of a Gough-Stewart platform as a surface defined over the field of rational functions on the the group of rotations. From the geometric properties of this surface we deduce, in the generic biplanar case, a rational parametrization and a family of parallel planes cutting the surface in the conics of a linear pencil, which are uniform for all generic orientations.

Keywords: Gough-Stewart platform, parallel singularities, cubic surface
\end{abstract}

\section{Introduction}

The aim of this paper is to contribute to the study of the singularity locus of a Gough-Stewart platform, which is a hypersurface Sing $\subset \mathrm{SE}(3)$ in the 6dimensional group of motions in 3 -space. This is a subject which has been addressed in many papers such as $[1,2,3,4,5,6,7,8,9]$. We consider in this paper the biplanar case, when the base and the platform are general planar hexagons. In particular, we address the problem of the rational parametrization of Sing.

It is mentioned in the review [1] that

A complete description and characterization of the singularities would be to parametrize the entire singularity hypersurface(s) in the task-space (6D in the case of the Gough-Stewart platform). ... Such a description is extremely difficult for the Gough-Stewart platform and, to the best of the authors' knowledge, no such work has been reported till now.

We do not know whether such a parametrization will actually prove to be useful. However, the mathematical study with tools of classical algebraic geometry that we use gives new insights on the geometry of the singularity locus Sing.

It is well known (see for instance [9]) that, if the orientation $R \in \mathrm{SO}(3)$ of the platform is fixed so that only translational part of the motion is left, then the singularity surface $\operatorname{Sing}_{R}$ in $\mathbb{R}^{3}$ is a cubic surface. When we let $R$ vary in $\mathrm{SO}(3)$, we obtain a family of cubic surfaces $\left(\mathrm{Sing}_{R}\right)_{R \in \mathrm{SO}(3)}$ parametrized by

${ }^{*}$ IRMAR, Université de Rennes 1, France

†Dép. de Mathématiques, Université de Maradi, Niger 
$\mathrm{SO}(3)$, whose total space is Sing. From the algebro-geometric point of view, this gives a cubic surface $\Sigma$ defined over the field $\mathbb{R}(\mathrm{SO}(3))$ of rational functions on $\mathrm{SO}(3)$ (this is the field generated over $\mathbb{R}$ by the coefficients $R_{i, j}$ of rotation matrices). The coefficients $R_{i, j}$ are not, of course, independant variables, but the field $\mathbb{R}(\mathrm{SO}(3))$ is isomorphic to the field of rational function in three variables $\mathbb{R}(u, v, w)$ (this can be seen using Cayley parametrization for rotations). The main part of this article is devoted to the study of the surface $\Sigma$. The reason for studying $\Sigma$ is that a property satisfied by $\Sigma$ is also satisfied by the surfaces $\operatorname{Sing}_{R}$ for generic orientation $R$, i.e. for all orientations $R$ outside a proper algebraic subset of $\mathrm{SO}(3)$.

In Section 2 we fix the notations and establish the equation of the singularity locus Sing in SE(3) for a general Gough-Stewart platform. The material in this section is already well known for the most part (see for instance [9]). However, the fact that the equation has degree 3 in the coefficients of the rotation matrix seems to be new.

From Section 3 onwards, we deal only with biplanar Gough-Stewart platforms. Section 3 is devoted to the study of the points at infinity of $\Sigma$. These points correspond to no actual pose of the platform, but they are responsible for remarkable geometric properties of the singularity surface. We prove that the part at infinity of $\Sigma$ is the union of three lines, two of which are the lines at infinity of the base plane and the mobile platform plane. We prove also that, for a generic architecture, the intersection of the latter two lines is the unique singular point of $\Sigma$ (more exactly, of its projective closure). We illustrate these facts with an example.

In Section 4 we use the singular point at infinity of $\Sigma$ to obtain a rational parametrization of $\Sigma$ with two parameters and coefficients in $\mathbb{R}(\mathrm{SO}(3))$. This in turn gives a rational parametrization of the singularity hypersurface Sing in $\mathrm{SE}(3)$ with five parameters and real coefficients. We also show that there is a family of parallel planes cutting $\Sigma$ in the conics of a linear pencil (this is different from the result in [8] where the conic sections do not belong to a linear pencil). We continue the example to illustrate these properties.

\section{Notations, and equation for the singularity lo- cus}

We consider in this section a Gough-Stewart platform with an arbitrary architecture. For $i=1, \ldots, 6$, we denote by $A_{i}$ the anchor point of the $i$-th leg on the base and by $B_{i}$ the anchor point on the mobile platform in the initial pose. We assume $A_{1}=B_{1}=(0,0,0)^{\mathbf{T}}$.

\subsection{Equation of the singularity locus in $S E(3)$}

We recall the derivation of the equation of the singularity hypersurface, mainly in order to fix notation. The computaton is very similar to the one in [9].

The group $S E(3)$ of rigid motion in 3-space acts on the mobile platform by the transformation $X \mapsto R X+P$ where $R$ is the rotation matrix and $P=$ $(x, y, z)^{\mathbf{T}}$ is the translation vector. So the coordinates of anchor points on the mobile platform are, after motion, $R B_{i}+P$ for $i=1 \ldots 6$. 
The Plücker coordinates of the legs are 6-dimensional vectors whose first three coordinates are $R B_{i}+P-A_{i}$ and last three coordinates (the moment with respect to the origin)

$$
A_{i} \times\left(R B_{i}+P-A_{i}\right)=A_{i} \times R B_{i}+A_{i} \times P .
$$

Since $A_{1}=B_{1}=0$, the Plücker coordinates of the first leg are given by $P$ and $(0,0,0)^{\mathbf{T}}$. For $i=2, \ldots, 6$ we set $E_{i}=R B_{i}-A_{i}$ and $F_{i}=A_{i} \times R B_{i}$.

It is well known and explained in [10] that the Gough-Stewart platform is in a singular configuration if and only if the Plücker coordinates of the six legs are linearly dependant. This is expressed by the vanishing of the $6 \times 6$ determinant of the matrix whose columns are the Plücker coordinates of the legs. This determinant is

$$
J=\operatorname{det}\left(\begin{array}{cccc}
P & E_{2}+P & \ldots & E_{6}+P \\
0 & F_{2}+A_{2} \times P & \ldots & F_{6}+A_{6} \times P
\end{array}\right)
$$

By subtracting the first column from the following ones, we obtain

$$
J=\operatorname{det}\left(\begin{array}{cccc}
P & E_{2} & \ldots & E_{6} \\
0 & F_{2}+A_{2} \times P & \ldots & F_{6}+A_{6} \times P
\end{array}\right)
$$

\subsection{Degree of the equation}

Theorem 1 The singularity locus Sing in $\mathrm{SE}(3)$ has an equation which is of degree 3 with respect to $P$ and also of degree 3 with respect to $R$. Moreover, the homogeneous part of degree 3 with respect to $P$ of the equation is of degree 2 with respect to $R$.

Proof: First, we proceed to show that the degree of $J$ w.r.t. $P$ is 3 . In order to do that, we write the $i$-th column (for $i \geq 2$ ) as the sum $\left(\begin{array}{c}E_{i} \\ F_{i}\end{array}\right)+\left(\begin{array}{c}0 \\ A_{i} \times P\end{array}\right)$ and develop the determinant $J$ using multilinearity. Notice that any three vectors $\left(\begin{array}{c}0 \\ A_{i} \times P\end{array}\right),\left(\begin{array}{c}0 \\ A_{j} \times P\end{array}\right)$ and $\left(\begin{array}{c}0 \\ A_{k} \times P\end{array}\right)$ are linearly dependant since they are all orthogonal to $P$ (or all 0 if $P$ is 0 ). So we obtain actually

$$
J=\operatorname{det}(L)+\sum_{i=2}^{6} \operatorname{det}\left(M_{i}\right)+\sum_{2 \leq i<j \leq 6} \operatorname{det}\left(N_{i, j}\right)
$$

where

$$
\text { - } L=\left(\begin{array}{cccc}
P & E_{2} & \ldots & E_{6} \\
0 & F_{2} & \ldots & F_{6}
\end{array}\right)
$$

- $M_{i}$ (for $\left.2 \leq i \leq 6\right)$ is the matrix whose first column is $\left(\begin{array}{c}P \\ 0\end{array}\right), i$-th column is $\left(\begin{array}{c}0 \\ A_{i} \times P\end{array}\right)$ and all other columns are $\left(\begin{array}{c}E_{k} \\ F_{k}\end{array}\right)$

- $N_{i, j}$ (for $2 \leq i<j \leq 6$ ) is the matrix whose first column is $\left(\begin{array}{l}P \\ 0\end{array}\right), i$-th and $j$-th columns are $\left(\begin{array}{c}0 \\ A_{i} \times P\end{array}\right)$ and $\left(\begin{array}{c}0 \\ A_{j} \times P\end{array}\right)$ and all other columns are $\left(\begin{array}{c}E_{k} \\ F_{k}\end{array}\right)$. 
We remark that $\operatorname{det}(L)$ is a homogeneous polynomial of degree 1 in $P$, every $\operatorname{det}\left(M_{i}\right)$ is a homogeneous polynomial of degree 2 in $P$ and every $\operatorname{det}\left(N_{i, j}\right)$ is homogeneous of degree 3 in $P$. Hence altogether $J$ is a polynomial of degree at most 3 in $P$ (this is rather well known and can be found for instance in [9]).

Now we determine the degree of $J$ w.r.t. $R$. We develop the expression of $J$ in equation (3) by writing each colum $\left(\begin{array}{c}E_{i} \\ F_{i}+A_{i} \times P\end{array}\right)$ as $\left(\begin{array}{c}E_{i} \\ 0\end{array}\right)+\left(\begin{array}{c}0 \\ F_{i}+A_{i} \times P\end{array}\right)$ and using multilinearity. We express the result using mixed products $[U, V, W]=$ $(U \times V) \cdot W$. We have

$$
\begin{aligned}
J= & \sum_{2 \leq i<j \leq 6}\left((-1)^{i+j-1}\left[P, R B_{i}-A_{i}, R B_{j}-A_{j}\right]\right. \\
& {\left.\left[A_{\widehat{i j}_{1}} \times R B_{\widehat{i j}_{1}}+G_{\widehat{i j}_{1}}, A_{\widehat{i j}_{2}} \times R B_{\widehat{i j}_{2}}+G_{\widehat{i j}_{2}}, A_{\widehat{i j}_{3}} \times R B_{\widehat{i j}_{3}}+G_{\widehat{i j} j_{3}}\right]\right), }
\end{aligned}
$$

where $\widehat{i j}_{1}<\widehat{i j}_{2}<\widehat{i j}_{3}$ are the indices between 2 and 6 except $i$ and $j$ and $G_{k}=A_{k} \times P$. When we develop the mixed product $\left[P, R B_{i}-A_{i}, R B_{j}-A_{j}\right]$ using multilinearity, we always get expressions of degre $\leq 1$ w.r.t. $R$. The only non-obvious case is dealt with

$$
\left[P, R B_{i}, R B_{j}\right]=\left[R^{-1} P, B_{i}, B_{j}\right]=\left[R^{\mathbf{T}} P, B_{i}, B_{j}\right]
$$

using the fact that rotation matrices preserve the mixed product. Similarly, when we develop $\left[A_{k} \times R B_{k}+G_{k}, A_{\ell} \times R B_{\ell}+G_{\ell}, A_{m} \times R B_{m}+G_{m}\right]$ using multilinearity, we always get expressions of degree $\leq 2$ w.r.t. $R$. The non-trivial case is dealt with

$$
\begin{aligned}
& {\left[A_{k} \times R B_{k}, A_{\ell} \times R B_{\ell}, A_{m} \times R B_{m}\right]} \\
& \quad=\left(\left(A_{k} \times R B_{k}\right) \times\left(A_{\ell} \times R B_{\ell}\right)\right) \cdot\left(A_{m} \times R B_{m}\right) \\
& \quad=\left[A_{k}, R B_{k}, R B_{\ell}\right]\left[A_{m}, R B_{m}, A_{\ell}\right]-\left[A_{k}, R B_{k}, A_{\ell}\right]\left[A_{m}, R B_{m}, R B_{\ell}\right] \\
& \quad=\left[R^{\mathbf{T}} A_{k}, B_{k}, B_{\ell}\right]\left[A_{m}, R B_{m}, A_{\ell}\right]-\left[A_{k}, R B_{k}, A_{\ell}\right]\left[R^{\mathbf{T}} A_{m}, B_{m}, B_{\ell}\right] .
\end{aligned}
$$

So we have shown that the degree of $J$ with respect to $R$ is at most 3 ; it is actually 3 .

We now deal with the homogeneous part $J_{\text {inf }}=\sum_{2<i<j<6} N_{i, j}$ of $J$ of degree 3 with respect to $P$ (the reason for the notation $J_{\text {inf }}$ will be clear in the next section). We develop $J_{\text {inf }}$ in the same way as we did for Equation (5) and so $J_{\text {inf }}$ is the sum of products $\left[P, R B_{k_{1}}-A_{k_{1}}, R B_{k_{2}}-A_{k_{2}}\right]\left[A_{i} \times P, A_{j} \times P, A_{k_{3}} \times R B_{k_{3}}\right]$ (with signs). We have already seen that the first mixed product is of degree at most 1 with respect to $R$, and the second mixed product is homogeneous of degree 1 with respect to $R$. It follows that $J_{\text {inf }}$ has degree at most 2 with respect to $R$.

\subsection{Cayley parametrization for rotation matrices}

Let $A=\left(\begin{array}{ccc}0 & -w & v \\ w & 0 & -u \\ -v & u & 0\end{array}\right)$ be a skew-symmetric matrix. Since 1 is not an eigenvalue of $A, I-A$ is invertible. The matrix $R=(I+A)(I-A)^{-1}$ is a 
rotation matrix

$$
R(u, v, w)=\frac{1}{\Delta}\left(\begin{array}{ccc}
1+u^{2}-v^{2}-w^{2} & 2(u v-w) & 2(u w+v) \\
2(u v+w) & 1-u^{2}+v^{2}-w^{2} & 2(v w-u) \\
2(u w-v) & 2(v w+u) & 1-u^{2}-v^{2}+w^{2}
\end{array}\right)
$$

where $\Delta=1+u^{2}+v^{2}+w^{2}$. If the vector $(u, v, w)^{\mathbf{T}}$ is not the zero vector, it spans the axis of the rotation $R$. The tangent of the half-angle of the rotation is $\sqrt{\Delta-1}$. The Cayley parametrization is a rational parametrization of all rotation matrices, except the half-turns. These half-turns are obtained as limits when $u^{2}+v^{2}+w^{2}$ tends to infinity; alternatively, one can use the homogeneous Euler-Rodrigues parametrization (that is, parametrization with quaternions) with one more variable.

Note that we can recover rationally $u, v, w$ from the rotation matrix $R(u, v, w)$. Indeed

$$
u=\frac{R_{3,2}-R_{2,3}}{1+\operatorname{tr}(R)}, v=\frac{R_{1,3}-R_{3,1}}{1+\operatorname{tr}(R)}, w=\frac{R_{2,1}-R_{1,2}}{1+\operatorname{tr}(R)},
$$

where $\operatorname{tr}(R)=R_{1,1}+R_{2,2}+R_{3,3}$. This shows:

Proposition 2 The Cayley parametrization induces an isomorphism between the field $\mathbb{R}(\mathrm{SO}(3))$ of rational functions on $\mathrm{SO}(3)$ and the field $\mathbb{R}(u, v, w)$ of rational function in three independant variables.

The preceding result says, in algebro-geometric terms, that the variety $\mathrm{SO}(3)$ is a rational variety over $\mathbb{R}$.

We shall need a technical result about Cayley parametrization:

Proposition 3 If a polynomial $S$ in $u, v, w$ is of degree $\leq 2 k$, then $\frac{S}{\Delta^{k}}$ can be expressed as a polynomial of degree $\leq k$ in $R_{i, j}$.

Proof: It suffices to check the property for monomials of degree $\leq 2$ in $u, v, w$. We have

$$
\begin{array}{lll}
\frac{1}{\Delta}=\frac{1}{4}(1+\operatorname{tr}(R)) & \frac{u}{\Delta}=\frac{1}{4}\left(R_{3,2}-R_{2,3}\right) \\
\frac{u^{2}}{\Delta}=\frac{1}{4}\left(1+R_{1,1}-R_{2,2}-R_{3,3}\right) & \frac{u v}{\Delta}=\frac{1}{4}\left(R_{1,2}+R_{2,1}\right)
\end{array}
$$

and, by circular permutation, similar formulas for the other momomials of degree $\leq 2$.

If we substitute the Cayley parametrization $R(u, v, w)$ for $R$ in $J$, we obtain a rational function of $(u, v, w)$. Multiplication by $\Delta^{3}$ chases the denominators, and we obtain now a polynomial equation in $x, y, z, u, v, w$ for the singularity locus, of degree 3 w.r.t. the translation variables $x, y, z$ and of degree 6 with respect to the Cayley parameters $u, v, w$.

\subsection{The cubic surface $\Sigma$}

We can view the equation $J=0$ as an equation in the three variables $x, y, z$ with coefficients in the field $\mathbb{R}(\mathrm{SO}(3))$ (or the field $\mathbb{R}(u, v, w)$, according to Proposition 
2). As such, this is the equation of a cubic surface $\Sigma$ defined over $\mathbb{R}(\operatorname{SO}(3))$. This surface $\Sigma$ will be the main object of study in the rest of this article. In the language of algebraic geometry, $\Sigma$ is the generic fiber of the family of surfaces $\operatorname{Sing}_{R}$ parametrized by $\mathrm{SO}(3)$. The idea is that the properties of $\Sigma$ will hold uniformly for all $\operatorname{Sing}_{R}$, except over a proper algebraic subset of $\mathrm{SO}(3)$. We shall illustrate this in what follows.

\section{The case of a biplanar platform: points at in- finity}

For the rest of the paper, we consider a Gough-Stewart platform such that the anchor points on the base are in a same plane $\Pi_{A}$ and the anchor points on the mobile platform are in a same plane $\Pi_{B}$ (depending on the motion in $\mathrm{SE}(3)$, of course). We call it a biplanar Gough-Stewart platform, which we abbreviate as BGSP. We assume that the base plane and the mobile platform plane coincide in the initial pose. We have an orthonormal frame with origin $A_{0}$ attached to the base (independant of the orientation $R$ ). We denote by $(x, y, z)$ the coordinates with respect to this frame, and we choose it so that the plane $\Pi_{A}$ has equation $z=0$. Let us call this frame the "canonical frame".

We investigate the singularity surface $\Sigma$ defined over the field $\mathbb{R}(\mathrm{SO}(3))$. The key to a better understanding of the geometry of this singularity surface is somewhat hidden: it lies in the consideration of its points at infinity. We denote by $\Sigma^{h}$ the projective closure of $\Sigma$. It is a cubic surface in the projective 3 -space over $\mathbb{R}(\mathrm{SO}(3))$. Its homogeneous equation, in the homogeneous variables $x, y, z, t$, is given by the homogeneization of $J$ :

$$
J^{h}(x, y, z, t)=t^{3} J\left(\frac{x}{t}, \frac{y}{t}, \frac{z}{t}\right) .
$$

The plane at infinity is the plane $t=0$, and the points at infinity of $\Sigma^{h}$ are the points with homogeneous coordinates $(x, y, z, 0)$ such that $J^{h}(x, y, z, 0)=0$, Note that $J_{\text {inf }}(x, y, z)=J^{h}(x, y, z, 0)$ is the homogeneous part of degree 3 of $J(x, y, z)$.

\subsection{Points at infinity of the singularity surface $\Sigma$}

The remarkable properties of the points at infinity of $\Sigma^{h}$ are described by the following theorems.

Theorem 4 The intersection of the projective singularity surface $\Sigma^{h}$ with the plane at infinity is the union of three lines, which are:

- the line at infinity of the base plane $\Pi_{A}$, with equations

$$
t=0, z=0
$$

- the line at infinity of the mobile platform plane $\Pi_{B}$, with equations

$$
t=0, R_{1,3} x+R_{2,3} y+R_{3,3} z=0 ;
$$


- another line at infinity with equations

$$
t=0, C_{1}(R) x+C_{2}(R) y+C_{3}(R) z=0,
$$

where $C_{1}, C_{2}, C_{3}$ are polynomials of degree at most 1 in the coefficients of the rotation matrix $R$.

These three lines are defined over $\mathbb{R}(\mathrm{SO}(3))$.

Proof: We homogenize the equation (4) of the singularity surface $\Sigma$ with the homogeneization variable $t$ in order to obtain the equation of the projective closure $\Sigma^{h}$ :

$$
J^{h}=t^{2} \operatorname{det}(L)+t \sum_{i=2}^{6} \operatorname{det}\left(M_{i}\right)+\sum_{2 \leq i<j \leq 6} \operatorname{det}\left(N_{i, j}\right) .
$$

The plane at infinity is the plane $t=0$, hence the intersection of $\Sigma^{h}$ with the plane at infinity is given by

$$
J_{\text {inf }}=\sum_{2 \leq i<j \leq 6} \operatorname{det}\left(N_{i, j}\right)=0
$$

We have already seen that $J_{\text {inf }}$ is the sum (with signs) of products of mixed products $\left[P, E_{k_{1}}, E_{k_{2}}\right]\left[A_{i} \times P, A_{j} \times P, F_{k_{3}}\right]$. If $z=0$, the third coordinates of $A_{i}$, $A_{j}$ and $P$ are all zero and it follows that $A_{i} \times P$ and $A_{j} \times P$ are collinear. Hence the mixed product $\left[A_{i} \times P, A_{j} \times P, F_{k_{3}}\right]$ vanishes and $J_{\text {inf }}=0$. This proves that $z$ divides $J_{\text {inf }}$. Recall that $z=0$ is the equation of the plane of the base. Since the base and the mobile platform have symmetric roles in the analysis of singularities, we conclude that $J_{\text {inf }}$ is also divisible by $R_{1,3} x+R_{2,3} y+R_{3,3} z$ which is the equation of the direction of the plane of the mobile platform. So we have found two linear factors of the cubic form $J_{\text {inf }}$ over $\mathbb{R}(\mathrm{SO}(3))$. Hence $J_{\text {inf factors as }}$

$$
J_{\mathrm{inf}}(x, y, z)=z\left(R_{1,3} x+R_{2,3} y+R_{3,3} z\right)\left(C_{1}(R) x+C_{2}(R) y+C_{3}(R) z\right),
$$

where the $C_{i}(R)$ belong to $\mathbb{R}(\mathrm{SO}(3))$. Since the $R_{i, j}$ are not independant variables we must take care in the discussion of the degree and use the independant variables $u, v, w$. We have seen that $J_{\text {inf }}$ has degree at most 2 with respect to $R$ i.e $\Delta^{2} J_{\text {inf }}$ has degree at most 4 with respect to $u, v, w$. Since $R_{1,3} x+R_{2,3} y+R_{3,3} z$ multiplied by $\Delta$ has degree 2 in $u, v, w$, we obtain that $\Delta C_{i}(R)$ has degree at most 2 in $u, v, w$. By Proposition $3, C_{i}$ of degree at most 1 in $R_{i, j}$. The theorem is proved.

Theorem 5 The point $P_{0}$, which is the point at infinity intersection of the line at infinity of the base plane $\Pi_{A}$ with the line at infinity of the mobile platform plane $\Pi_{B}$, is a singular point of the projective singularity surface $\Sigma^{h}$. This singular point is defined over $\mathbb{R}(\mathrm{SO}(3))$ and has homogeneous coordinates $\left(R_{2,3},-R_{1,3}, 0,0\right)$. It is the only singular point of $\Sigma^{h}$ for a generic BGSP.

Proof: We prove the theorem by brute force, computing $J$ in a direct orthonormal frame different from the canonical frame, with the aid of a computer algebra software. This frame has origin $A_{0}$ and basis $(\vec{i}, \vec{j}, \vec{k})$ such that the first vector $\vec{i}$ 
gives the common direction of $\Pi_{A}$ and $\Pi_{B}$ and the first two vectors $\vec{i}, \vec{j}$ generate the direction of the base plane $\Pi_{A}$. Specifically, the normal to the base plane is directed by $\vec{k}=(0,0,1)^{\mathbf{T}}$ and the normal to the mobile platform plane is directed by the third column of the rotation matrix $R$, say $\vec{n}_{B}(R)=\left(R_{1,3}, R_{2,3}, R_{3,3}\right)^{\mathbf{T}}$. So we can take

$$
\begin{aligned}
& \vec{i}=\frac{1}{\left\|\vec{n}_{B} \times \vec{k}\right\|} \vec{n}_{B} \times \vec{k}=\frac{1}{\sqrt{N}}\left(R_{2,3},-R_{1,3}, 0\right)^{\mathbf{T}}, \\
& \vec{j}=\frac{1}{\sqrt{N}}\left(R_{1,3}, R_{2,3}, 0\right)^{\mathbf{T}},
\end{aligned}
$$

where $N=\left(R_{1,3}^{2}+R_{2,3}^{2}\right)$ is a non-zero element of the field $\mathbb{R}(\mathrm{SO}(3))$. So this orthonormal frame is actually not defined over the field $\mathbb{R}(\mathrm{SO}(3))$, but on its extension $\mathbb{R}(\mathrm{SO}(3))[\sqrt{N}]$ by the square root of $N$. Note that the direction of the plane $\Pi_{B}$ is generated by $\vec{i}$ and $\vec{n}_{B} \times \vec{i}=R_{3,3} \vec{j}-\sqrt{N} \vec{k}$. We have $R_{3,3}^{2}+N=1$, and we set $c=R_{3,3}, s=-\sqrt{N}$ for short.

In this chosen frame, the points $A_{i}$ have coordinates $\left(\lambda_{i}, \mu_{i}, 0\right)^{\mathbf{T}}$ and the points $R B_{i}$ have coordinates $\left(\nu_{i}, c \rho_{i}, s \rho_{i}\right)^{\mathbf{T}}$ for $i=2, \ldots, 6$ (all $\lambda_{i}, \mu_{i}, \nu_{i}, \rho_{i}$ are actually elements of the field $\mathbb{R}(\mathrm{SO}(3))[\sqrt{N}]$ which we do not make explicit). We denote by $\left(x^{\prime}, y^{\prime}, z\right)$ the coordinates of $P$ in this frame. We compute $J$ according to formula (3), keeping $\lambda_{i}, \mu_{i}, \nu_{i}, \rho_{i}, c, s$ variable, and homogenize the result with respect to $x^{\prime}, y^{\prime}, z$ to $J^{h}$ with homogeneization variable $t$.

The point $P_{0}$ has homogeneous coordinates $\left(x^{\prime}, y^{\prime}, z, t\right)=(1,0,0,0)$ with respect to the chosen frame. The computation shows that the four partial derivatives (with respect to $x^{\prime}, y^{\prime}, z, t$ ) of $J^{h}$ all evaluate to 0 at this point. This implies that $P_{0}$ is a singular point of the projective cubic surface $\Sigma^{h}$. Since the lines at infinity of $\Pi_{A}$ and $\Pi_{B}$ are defined over $\mathbb{R}(\operatorname{SO}(3))$, this is also the case for their intersection point $P_{0}$; we can take $\left(R_{2,3},-R_{1,3}, 0,0\right)$ as homogeneous system of coordinates for $P_{0}$ in the canonical frame.

In order to prove that $P_{0}$ is the only singular point of $\Sigma^{h}$ for generic BGSP, it is sufficient to prove this in an example, for the projective closure $\operatorname{Sing}_{R}^{h}$ of the singularity surface $\operatorname{Sing}_{R}$ for a chosen orientation $R$. Indeed the set of $R \in \mathrm{SO}(3)$ for which $\mathrm{Sing}_{R}^{h}$ has more singular points (real or complex) than just $P_{0}(R)$ is an algebraic subset of $\mathrm{SO}(3)$. It suffices to show that this algebraic subset is a proper subset in order to conclude that generically $\operatorname{Sing}_{R}^{h}$ has no other singular point than $P_{0}(R)$, which means that $\Sigma^{h}$ has no other singular point than $P_{0}$. We shall check this fact in the example of the next subsection.

We have proved that $\Sigma^{h}$ is, for a generic BGSP, a cubic surface with a unique singular point. Actually it corresponds to the second of the twenty three cases of cubic surfaces in the classification contained in the memoir of A. Cayley [11]. Indeed it can be checked that the unique singular point has a nondegenerate tangent cone.

\subsection{An example}

We compute an example with $A_{1}=(0,0,0), A_{2}=(1,-1,0), A_{3}=(2,0,0)$, $A_{4}=(2,1,0), A_{5}=(1,1,0), A_{6}=(0,1,0), B_{1}=(0,0,0), B_{2}=(1,-1,0)$, $B_{3}=(2,-1,0), B_{4}=(3,0,0), B_{5}=(2,1,0), B_{6}=(1,1,0)$ (see Figure 1, left). 

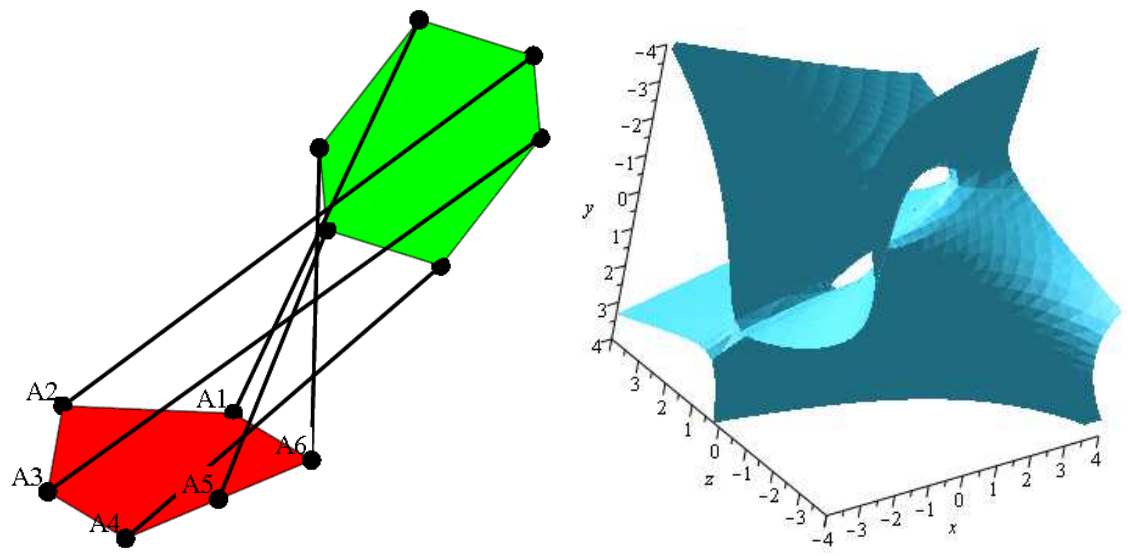

Figure 1: The BGSP and its singularity surface for the orientation of the example

We perform the computation using a rotation matrix with coefficients $R_{i, j}$ with $1 \leq i, j \leq 3$.

The computation of the equation $J$ of the surface of singularities as explained in subsection 2.2 gives us a polynomial of degree 3 in the position variables $x, y, z$ and also of degree 3 in the orientation coefficients $R_{i, j}$. The homogeneous part $J_{\text {inf }}$ of degree 3 with respect to $x, y, z$ of $J$ factors as the product of three linear forms, as proved in Theorem 4:

$$
\begin{aligned}
& J_{\mathrm{inf}}=z \times\left(R_{1,3} x+R_{2,3} y+R_{3,3} z\right) \times \\
& \left(\left(R_{3,2}-2 R_{3,1}\right) x+2\left(R_{3,2}+R_{3,1}\right) y+\left(2 R_{1,1}-2 R_{2,1}-2 R_{2,2}-R_{1,2}\right) z\right)
\end{aligned}
$$

The first two factors correspond to the direction of the base plane $\Pi_{A}$ and of the mobile platform plane $\Pi_{B}$. The remaining part of $J$ is a polynomial of degree 2 with respect to $x, y, z$, without constant term, whose coefficients are polynomials of degree 3 in the $R_{i, j}$. If we evaluate at the following rotation matrix, corresponding to Cayley parameters $u=1, v=1, w=-3 / 2$ (and to the orientation of Figure 1, left):

$$
R=\frac{1}{21}\left(\begin{array}{ccc}
-5 & 20 & -4 \\
-4 & -5 & -20 \\
-20 & -4 & 5
\end{array}\right),
$$

we get the following equation for the singularity surface, clearing denominators:

$$
\begin{aligned}
21 z(4 x+20 y-5 z)(-3 x+4 y+z)+352 x^{2}+1968 x y+1040 y^{2} \\
+1312 x z-236 y z-2736 z^{2}-608 x+160 y-216 z=0
\end{aligned}
$$

The surface is represented in Figure 1, right. We look for singular points of the projective closure of this cubic surface, using a computer algebra software. We find that the only singular point is the point with homogeneous coordinates $(-5,1,0,0)$, that is the point $P_{0}$ of Theorem 5 . The fact that there is no other singular point finishes the proof of Theorem 5 . 


\section{Rational parametrization and pencil of conics}

\subsection{Rational parametrization in the biplanar case}

Theorem 4 establishes that the homogeneous part of degree 3 of $J(x, y, z)$ factors into the product of three linear forms $L_{1}=z, L_{2}=R_{1,3} x+R_{2,3} y+R_{3,3} z$ and $L_{3}=C_{1}(R) x+C_{2}(R) y+C_{3}(R) z$ where the coefficients $C_{i}(R)$ are polynomials of degree 1 in the coefficients of the rotation matrix $R$. We perform a linear change of variables, defined over the field $\mathbb{R}(\mathrm{SO}(3))$, from the variables $(x, y, z)$ to new position variables $(p, q, r)$ by choosing a (non-orthonormal) basis $\left(\vec{e}_{1}, \vec{e}_{2}, \vec{e}_{3}\right)$ such that $\left(L_{1}, L_{2}, L_{3}\right)$ is the dual basis of $\left(\vec{e}_{1}, \vec{e}_{2}, \vec{e}_{3}\right)$, i.e. the $L_{i}$ are the coordinate forms in the new basis $\left(\vec{e}_{1}, \vec{e}_{2}, \vec{e}_{3}\right)$. Concretely we have

$$
\left(\begin{array}{l}
x \\
y \\
z
\end{array}\right)=\Lambda^{-1}\left(\begin{array}{l}
p \\
q \\
r
\end{array}\right) \text {, where } \Lambda=\left(\begin{array}{ccc}
0 & 0 & 1 \\
R_{1,3} & R_{2,3} & R_{3,3} \\
C_{1}(R) & C_{2}(R) & C_{3}(R)
\end{array}\right) .
$$

The $i$-th line of the matrix $\Lambda$ is made of the coefficients of the linear form $L_{i}$. This matrix $\Lambda$ is invertible over the field $\mathbb{R}(\mathrm{SO}(3))$ for a generic BGSP. Indeed, $\operatorname{det}(\Lambda)=R_{1,3} C_{1}(R)-R_{2,3} C_{2}(R)$ is a non zero element of $\mathbb{R}(\mathrm{SO}(3))$ for a generic BGSP. Substituting $x, y, z$ for their expressions in terms of $p, q, r$ in the equation $J(x, y, z)$ of $\Sigma$, we obtain an equation $\tilde{J}(p, q, r)$ of degree 3 in the variables $p, q, r$, whose coefficients are elements of $\mathbb{R}(\mathrm{SO}(3))$, i.e. rational functions in the $R_{i, j}$.

According to the choice of the new variables $p, q, r$, the homogeneous part of degree three of the equation $\tilde{J}(p, q, r)$ is simply the product $p q r$. It can be written as

$$
\tilde{J}(p, q, r)=p q r+Q(p, q, r)+S(p, q, r)
$$

where $Q$ is a quadratic form and $S$ a linear form both with coefficients in $\mathbb{R}(\mathrm{SO}(3))$ (there is no constant term since the origin belongs to $\Sigma$ ). The following theorem gives a rational parametrization of $\Sigma$ in a simple form.

Theorem 6 The equation $\tilde{J}(p, q, r)$ of the singularity surface $\Sigma$ can be written as

$$
\tilde{J}(p, q, r)=r \Gamma_{1}(p, q)+\Gamma_{0}(p, q)
$$

where $\Gamma_{1}(p, q)$ and $\Gamma_{0}(p, q)$ are polynomial of degree 2 with coefficients in $\mathbb{R}(\mathrm{SO}(3))$ (i.e. rational functions of the $R_{i, j}$ ). Moreover, the quadratic part of $\Gamma_{1}$ is $p q$.

Hence, the surface $\Sigma$ has the rational parametrization $r=-\Gamma_{0}(p, q) / \Gamma_{1}(p, q)$ over $\mathbb{R}(\mathrm{SO}(3))$.

Proof: Considering the expression of $\tilde{J}$ written in $(20)$, it suffices to prove that the coefficient $Q_{3,3}$ of $r^{2}$ in the quadratic form $Q(p, q, r)$ is zero. This will turn out to be a consequence of the existence of a singular point at infinity of the projective surface $\Sigma^{h}$. The homogeneous equation of this projective surface, in the homogeneous coordinates $(p, q, r, t)$, is written as

$$
\tilde{J}^{h}(p, q, r, t)=p q r+Q(p, q, r) t+S(p, q, r) t^{2} .
$$

The singular point described in Theorem 5 has homogeneous coordinates $(0,0,1,0)$ in the new system of coordinates (according to the choice of $p, q, r$ ). Hence, all partial derivatives of $\tilde{J}^{h}(p, q, r, t)$ must vanish at $(0,0,1,0)$. The value of $\partial \tilde{J}^{h} / \partial t$ at $(0,0,1,0)$ is precisely the coefficient $Q_{3,3}$ of $r^{2}$ in $Q$. Hence $Q_{3,3}=0$, which proves the theorem. 
Corollary 7 Sing $\subset \mathrm{SE}(3)$ has a rational parametrization with five parameters $p, q, u, v, w$ and coefficients in $\mathbb{R}$.

Proof: The coefficient of $R \in \mathrm{SO}(3)$ are rational functions of the Cayley parameters $u, v, w$. Replacing the $R_{i, j}$ with these rational functions of $u, v, w$ in $r=-\Gamma_{0}(p, q) / \Gamma_{1}(p, q)$, we obtain $r$ as a rational function of $p, q, u, v, w$ with real coefficients. Using $\left(\begin{array}{l}x \\ y \\ z\end{array}\right)=\Lambda^{-1}\left(\begin{array}{l}p \\ q \\ r\end{array}\right)$ and replacing the $R_{i, j}$ in $\Lambda^{-1}$ with rational expression in function of $u, v, w$, we obtain $x, y, z$ as rational function of $p, q, u, v, w$ with real coefficients.

Remark that this parametrization is uniformly well defined for all orientations $R \in \mathrm{SO}(3)$, except those for which $\operatorname{det}(\Lambda)=R_{1,3} C_{1}(R)-R_{2,3} C_{2}(R)=0$ which form a proper algebraic subset of $S O(3)$ for a generic $B G S P$. Among these orientations are those for which the plane of the mobile platform is parallel to the base plane (in this case the singularity surface for a generic BGSP is a triple plane), but there are more: those for which the three lines at infinity are distinct and concurrent. In these latter cases, the cubic surface $\operatorname{Sing}_{R}$ has still only one singular point at infinity and the tangent cone at this point degenerates in two planes; this is the third case in Cayley's classification [11].

\subsection{Conics on the singularity surface}

If a line $\ell$ is contained in a cubic surface, any plane containing $\ell$ cuts the cubic surface in the union of $\ell$ with a conic. Since we have three lines at infinity in the singularity surface $\Sigma^{h}$, this gives three families of parallel planes cutting conics on $\Sigma$; indeed, the planes containing a line at infinity form a family of parallel planes having in common this line at infinity.

The first family is the family of planes parallel to the base plane $\Pi_{A}$, which have equations $z=$ constant (in the $x, y, z$ system of coordinates) or $p=$ constant (in the $p, q, r$ system of coordinates). Since the singular point of $\Sigma^{h}$, with homogeneous coordinates $(0,0,1,0)$ in the $p, q, r, t$ homogeneous coordinate system, is a point at infinity of all these planes, the conic cut on $\Sigma$ by any of these planes has this point as point at infinity; this means that all these conics are hyperbolas with an asymptote parallel to the $r$-axis of the $p, q, r$ coordinate system. This phenomenon was observed in [8], without a complete theoretical justification. It follows also from the general theory of cubic surfaces (see for instance [11]) that there are five planes through the line at infinity $z=t=0$ (or $p=t=0$ ) for which the intersection with $\Sigma^{h}$ is a union of 3 lines ; since the plane at infinity of the projective space is one of them, there are four planes $z=$ constant cutting the singularity surface $\Sigma$ in two lines, with one of these lines parallel to the $r$-axis. This confirms the observation made in [8]. For a general choice of the orientation $R$, two of four (not zero) of these planes may be real.

The second family is the family of planes parallel to the platform plane $\Pi_{B}$, which have equations $R_{1,3} x+R_{2,3} y+R_{3,3} z=$ constant (in the $x, y, z$ system of coordinates) or $q=$ constant (in the $p, q, r$ system of coordinates). Due to the symmetry between the base plane and the mobile platform plane, the analysis is exactly the same as for the first family. 
The most interesting is the third family, that is the family of planes with equations $C_{1}(R) x+C_{2}(R) y+C_{3}(R) z=$ constant (in the $x, y, z$ system of coordinates) or $r=$ constant (in the $p, q, r$ system of coordinates). According to Theorem 6 and the form of the equation of $\Sigma$ in the $p, q, r$ coordinates, the sections of $\Sigma$ by these planes are the conics of the linear pencil of conics in the plane $(p, q)$ generated by the two conics $\Gamma_{0}(p, q)=0$ and $\Gamma_{1}(p, q)=0$. All conics in this linear pencils are obtained as sections, except the conic $\Gamma_{1}(p, q)=0$ corresponding to $r=\infty$; note that this conic is a hyperbola since the quadratic part of its equation is $p q$. There are three planes $r=$ constant for which the conic degenerates in the union of two lines (the plane at infinity count here with multiplicity 2 , so that altogether we have the five planes from the general theory). For a general choice of orientation $R$, one or three of these planes may be real.

\subsection{Example continued}

We continue with the example of subsection 3.2. The formula 19 specializes as

$$
\begin{aligned}
& x=\left[\left(R_{2,3}\left(-2 R_{2,2}-R_{1,2}-2 R_{2,1}+2 R_{1,1}\right)-R_{3,3}\left(2 R_{3,1}-2 R_{3,2}\right)\right) p\right. \\
& \left.+\left(2 R_{3,1}+2 R_{3,2}\right) q-R_{2,3} r\right] / \delta, \\
& y=\left[\left(R_{1,3}\left(2 R_{2,2}+R_{1,2}+2 R_{2,1}-2 R_{1,1}\right)-R_{3,3}\left(2 R_{3,1}+R_{3,2}\right)\right) p\right. \\
& \left.+\left(2 R_{3,1}-R_{3,2}\right) q+R_{1,3} r\right] / \delta, \\
& z=p \text {, } \\
& \text { where } \delta=R_{1,3}\left(2 R_{3,1}+2 R_{3,2}\right)+R_{2,3}\left(2 R_{3,1}-R_{3,2}\right) \text {. }
\end{aligned}
$$

Then the rational parametrization of Theorem 6 is:

$$
r=\frac{\beta_{2,0} p^{2}+\beta_{1,1} p q+\beta_{0,2} q^{2}+\beta_{1,0} p+\beta_{0,1} q}{p q+\alpha_{1,0} p+\alpha_{0,1} q+\alpha_{0,0}},
$$

where the $\alpha_{k, \ell}$ and $\beta_{k, \ell}$ are rational functions of the $R_{i, j}$ with denominator $\delta$. For instance

$$
\begin{aligned}
\alpha_{0,1}=( & -3 R_{1,3} R_{3,2} R_{3,1}-3 R_{2,3} R_{3,1} R_{3,2}-2 R_{3,1}^{2} R_{2,3}+4 R_{3,2}^{2} R_{1,3} \\
& \left.-R_{3,2}^{2} R_{2,3}+2 R_{3,1}^{2} R_{1,3}\right) / \delta
\end{aligned}
$$

but this is one of the simplest coefficients. Using Cayley parameters $u, v, w$ and chasing denominators, we obtain a parametrization of the same form as (22), but with coefficients of degree 8 with respect to $u, v, w$; this shows, according to Proposition 3 , that the $\alpha_{k, \ell}$ and $\beta_{k, \ell}$ have expressions of degree at most 4 with respect to $R_{i, j}$.

Now we fix an orientation corresponding to Cayley parameters $u=1, v=$ $1, w=-3 / 2$, as in subsection 3.2. The formula (21) for the change of variables gives

$$
x=\frac{10}{19} p-\frac{21}{19} q+\frac{35}{76} r, \quad y=\frac{11}{76} p-\frac{63}{76} q-\frac{7}{76} r, \quad z=p
$$

and the parametrization (22) specializes as

$$
r=\frac{12\left(1638 p^{2}+2793 p q-2667 q^{2}+464 p-488 q\right)}{8379 p q+8736 p-8148 q-3200} .
$$



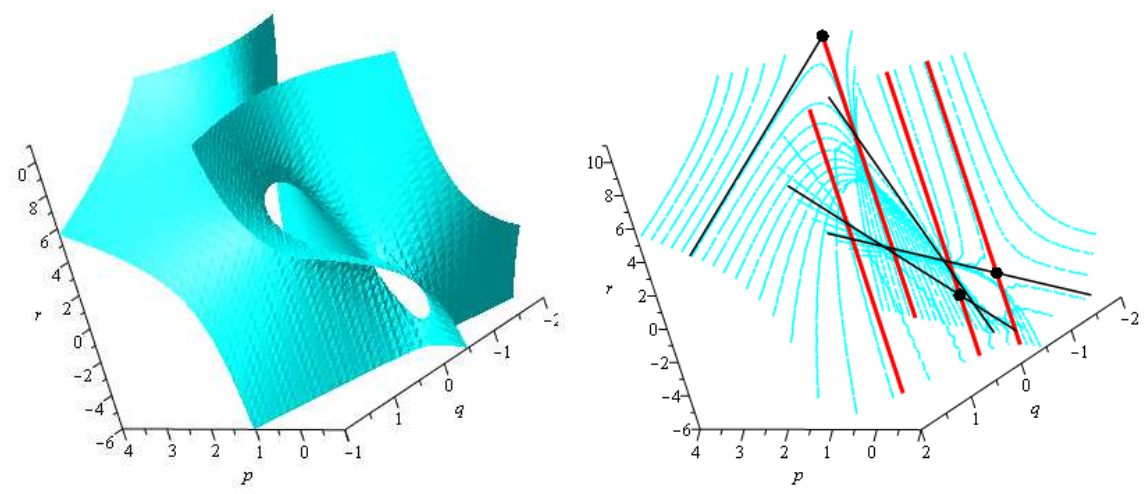

Figure 2: Singularity surface in $p, q, r$ coordinates and hyperbolas cut by planes $p=$ const

The figure 2 represents the singularity surface in the $p, q, r$ coordinates and its sections by planes $p=$ constant, which are all hyperbolas except for four sections which degenerate in two lines, one of them (in red) parallel to the $r$ axis, in direction of the singular point at infinity. The figure 3 represents the sections by planes $r=$ const. These sections are the conics of a linear pencil whose base points are the intersections with the red lines already encountered, which is clear on the view at the right. The pencil contains three degenerate conics represented in black on the left view.
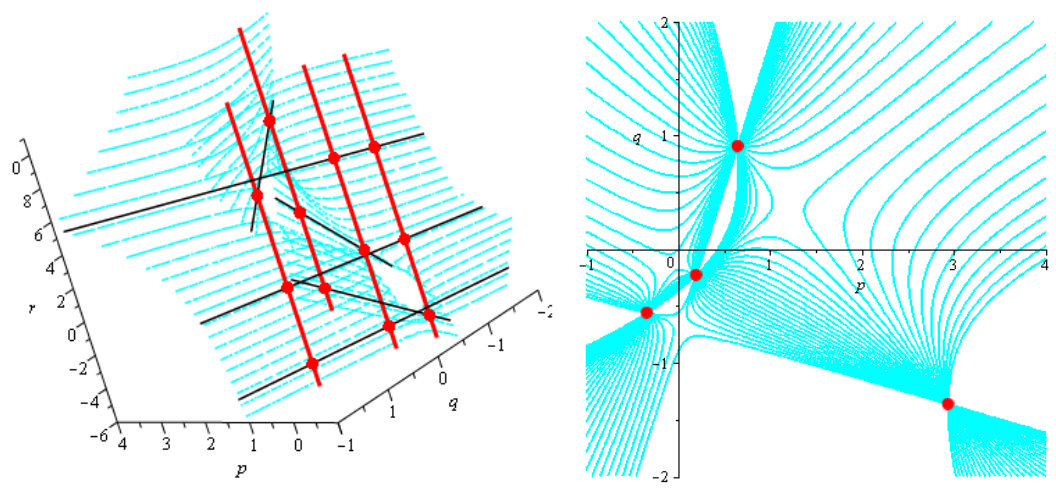

Figure 3: Conics cut by planes $r=$ const form a linear pencil

\section{Conclusion}

Many papers have already studied the locus of singularities of a Gough-Stewart platform. In this paper we proposed 
- to work as much as possible over the field $\mathbb{R}(\mathrm{SO}(3))$ in order to obtain results which are uniform for all orientations except a proper algebraic subset of the the group of rotations,

- to use some tools of algebraic geometry such as the consideration of points at infinity or the classical results of the theory of projective cubic surfaces.

As a result of this approach we obtained a uniform rational parametrization of the singularity locus, valid for generic orientations. We also exhibited a uniform family of parallel planes cutting the singularity surface in the conics of a linear pencil.

We plan to use the information obtained on the geometry of the locus of singularities to address other problems such as the number of aspects for a generic biplanar Gough-Stewart platform. We shall also study the geometry of the singularity locus when the Gough-Stewart platform is not assumed biplanar; in this general case, the famous configuration of the 27 lines (see [11]) on the surface $\Sigma$ plays a crucial role.

\section{References}

[1] B. Dasgupta, T.S. Mruthyunjaya: The Stewart platform manipulator: a review. Mechanism and Machine Theory 35 (2000) 15-40

[2] Y. Cao, Z. Huang, H. Ding and H. Zhou: Orientation-Singularity and Nonsingular orientation Workspace Analysis of the 6-6 Stewart Manipulator. Chin. J. Mech. Eng., 41 (2005), 50-55

[3] F. Pernkopf and M. L. Husty: Workspace analysis of Stewart-Goughtype parallel manipulators. Journal of Mechanical Engineering Science, 220 (2006), 1019-1032

[4] A. Karger: Stewart-Gough platforms with simple singularity surface. In Advances in Robot Kinematics - Mechanisms and Motion (J. Lenarcic, J. and B. Roth, eds), pages 247-254, Springer, 2006

[5] G. Nawratil: Results on Planar Parallel Manipulators with Cylindrical Singularity Surface. In Advances in Robot Kinematics - Analysis and Design (J. Lenarcic and P. Wenger, eds.), pages 321-330, Springer, 2008

[6] J. Borràs, F. Thomas and C. Torras: Singularity-Invariant Leg Rearrangements in Stewart-Gough Platforms. In Advances in Robot Kinematics Motion in Man and Machine, (J. Lenarčič and M. Stanisic, eds), pages 421-428, Springer, 2010.

[7] G. Nawratil: Stewart Gough platforms with non-cubic singularity surface. Mech. Mach. Theory, 45 (2010), 1851-1863

[8] V. Shanker, S. Banyopadhyay: Singular Manifold of the General Hexagonal Stewart Platform Manipulator. In Latest Advances in Robot Kinematics (J. Lenarčič and M. Husty, eds), pages 397-404, Springer, 2012 
[9] K. Doyon, C. Gosselin and P. Cardou: A frame-independant vector expression of the singularity locus of the Gough-Stewart platform. Proceedings of the ASME Mechanisms and Robotics Conference, Chicago, USA, 12-15 august 2012, paper DETC2012-70526.

[10] J-P. Merlet: Parallel Robots. Springer 2006.

[11] Cayley: A Memoir on Cubic Surfaces. Philosophical Transactions of the Royal Society of London, 159 (1869), 231-326 